\title{
Elevation Angle Estimation by Time Reversal Method in Homogeneous Shallow Sea Environment
}

\author{
Haixia Jing ${ }^{1,2}$, Haiyan Wang ${ }^{1, *}$, Zhengguo Liu $^{3}$, Xiaohong Shen ${ }^{1}$ and Tianyi Jia ${ }^{1}$ \\ ${ }^{1}$ School of Marine Science and Technology, Northwestern Polytechnic University, Xi'an Shaanxi 710072, China \\ ${ }^{2}$ College of Engineering, Xi'an International University, Xi' an Shanxi 710077, China \\ ${ }^{3}$ China Shipbuilding Industry Corporation, Beijing, 100097, China \\ ${ }^{*}$ Corresponding author
}

\begin{abstract}
This article discusses the problem of active detection and estimation for the elevation angle of a target in a homogeneous shallow sea environment, where a method using active detection on virtual time reversal (ADVTR) is proposed to solve the estimator performance decline problem caused by multipath and clutter. Unlike the conventional passive target estimation method ignoring the multipath effect, the ADVTR method will take full account of the multipath which exploits active detection (AD) to estimate channel, and virtual time reversal (VTR) to realize focusing in a computer to increase the signal-to-noise ratio (SNR) after the source-receive array (SRA) receives the reflected signal of the target. Furthermore, the Capon algorithm is adopted to compare the performance of ADVTR estimator and conventional one. Numerical simulations demonstrate its superior performance than the conventional estimators.
\end{abstract}

Keywords-active detection on virtual time reversal (ADVTR); elevation angle estimation; multipath effects; ULA; Capon algorithm

\section{INTRODUCTION}

To locate the position of a target, the range, azimuth and elevation angle need to be calculated simultaneously. Direction of arrival (DOA) estimation (including the horizontal and vertical plane) plays an important role in array signal processing with wide applications in communication, sonar and radar systems [1]. In this paper, we will focus on the DOA estimation of vertical plane (or the elevation angle estimation) in homogeneous shallow sea environment.

One of the important features in homogeneous shallow sea environment is the multipath effect of the channel. The conventional array signal processing DOA estimation method is based on the direct-path-only assumption; in which multipath is ignored or treated as a negative influence so as to be eliminated [2].

Time reversal [3] (TR) signal processing method raised in recent years, on the contrary, is to take full advantage of the multipath effect and the adaptive focusing characteristics to eliminate the problem of signal propagation channel caused by the multipath, which provides a new idea and method for highprecision DOA estimation.

TR can be divided into active time reversal (ATR) and passive time reversal (PTR) according to the implementation mode. The main difference is that it is necessary to re-emit the received signal with the noise to the real channel to complete the focusing for ATR after time reversal mirror (TRM) receiving the reflected signal from the target, but for the PTR, TR operation and focusing are completed in the virtual channel established by simulation software in computers [4]. PTR is also referred as to virtual time reversal (VTR) in [5]. Combining the advantages of ATR with PTR, the first process of ADVTR will realize the detection and channel estimation in the real channel and the second process will complete TR focusing virtually in the computer. Compared with ATR, there is no real re-emitting process so that the second receiving process has no noise in ADVTR and the signal-to-noise ratio (SNR) of the array received signal are higher. Compared with PTR, the characteristics of ocean channel can been estimated ideally according to the active detection while avoiding the PTR approximation process on the basis of the same processing gain with PTR. Based on this idea, the ADVTR method can be applied to DOA estimation in active acoustic array to achieve better performance.

There are some literatures on the DOA estimation using ATR and PTR. For example, Publication [6] employs ATR for the DOA estimation of a passive target in radar system, and combines the TR/range estimator to realize the active array source location. Publication [7] proposes a VTR method for passive direction of arrival estimation of electromagnetic signal emitted from a single communication station. Publication [8] addresses a DOA estimation method with PTR in a low angle target parameter estimation and antenna array scenario. Publication [9] utilizes PTR to study the orientation estimation performance of underwater targets, which proposes a PTR super-directional element distribution model based on nonuniform linear array (NLA). Above methods have verified the validness of TR methods in improving the performance of DOA estimator under multipath conditions.

Relative to [6-9], the target DOA estimation method in vertical plane based on ADVTR is introduced from array signal processing DOA estimation in this paper. The ADVTR multipath model based on uniform line array (ULA) is established, and Capon algorithm is applied to achieve target DOA estimation. Armed with the acoustic field simulation tool Bellhop, the experimental data are obtained and the model is simulated. The performances of the estimation algorithm with and without ADVTR are compared. 


\section{Multipath DOA Estimation MOdEL FOR ULA BASED ON ADVTR}

\section{A. Multipath Model Diagram for ULA}

The multipath DOA estimation model diagram for ULA based on the ray theory is shown in Figure 1.

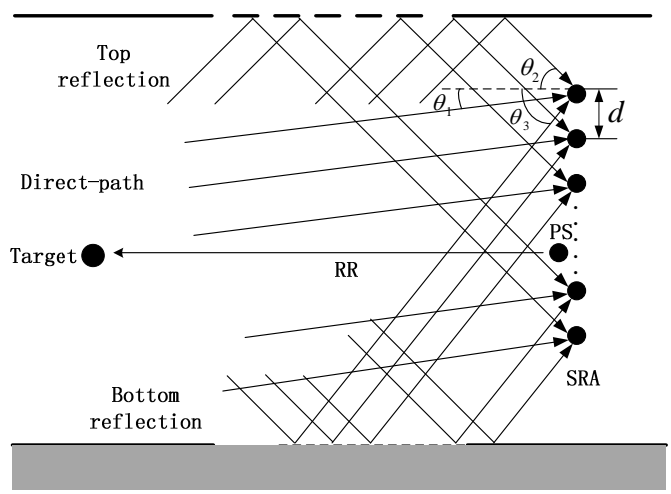

FIGURE I. MULTIPATH DOA ESTIMATION DIAGRAM FOR ULA

SRA in the Figure 1 is the vertical ULA, whose element number is $P$ and interelement spacing is $d$. For convenience, only three propagation paths between the target and SRA are drawn: the direct-path, the top-reflection-path and the bottomreflection-path, whose incident angle (i.e. DOA of backscatter from target propagating via various paths) is as follows: $\theta_{1}, \theta_{2}$ and $\theta_{3}$. To be more general, the acoustic propagation paths deduced subsequently are not confined to these three paths, but $N$ or $M$.

\section{B. Conventional Multipath DOA Model for ULA}

PS located in element $k$ of SRA will transmit the signal $f(t)$. According to the ray theory, the channel transfer function between the transmitting acoustic source and the target can be expressed as

$$
h_{k}(t)=\sum_{n=1}^{N} c_{k n} \delta\left(t-\tau_{k n}\right)
$$

Where $N$ is the total number of sound ray; $C_{k n}$ is the amplitudes (or attenuation) of n-th eigen ray (also regarded as the propagation path) from element $k$ to target; and $\tau_{k n}$ is the travel time (or delay) of n-th eigen ray from element $k$ to target.

Ignoring the effect of the target reflection, the signal received by the element $j$ is:

$$
\begin{aligned}
y_{j}(t) & =\left(f(t) \otimes h_{k}(t)\right) \otimes h_{j}(t)+v_{j}(t) \\
& =\sum_{m=1}^{M} \sum_{n=1}^{N} c_{j m} c_{k n} f\left(t-\tau_{j m}-\tau_{k n}\right)+v_{j}(t)
\end{aligned}
$$

Where $h_{j}(t)$ represents the channel function between the target and element $j ; v_{j}(t)$ is the noise between the element $j$ and the target; $c_{j m}$ and $\tau_{j m}$ represent amplitudes and travel time of the m-th propagation path from target to element $j$ separately.

Suppose the transmitter signal as $f(t)=s(t) e^{j \omega_{c} t}$. Combining far field and narrow band model theory of array signal processing, Equation (2) can be expressed as:

$$
\begin{aligned}
y_{j}(t) & =\sum_{m=1}^{M} \sum_{n=1}^{N} c_{j m} c_{k n} f\left(t-\tau_{k n}-\tau_{1 m}-\Delta \tau_{j m}\right)+v_{j}(t) \\
& \approx \sum_{m=1}^{M} \sum_{n=1}^{N} c_{j m} c_{k n} s(t) e^{j \omega_{c}\left(t-\tau_{k n}-\tau_{1 m}-\Delta \tau_{j m}\right)}+v_{j}(t) \\
& =\sum_{m=1}^{M} c_{j m} e^{-j \omega_{c} \Delta \tau_{j m}} e^{-j \omega_{c} \tau_{1 m}} \cdot \sum_{n=1}^{N} c_{k n} e^{-j \omega_{c} \tau_{k n}} \cdot s(t) e^{j \omega_{c} t}+v_{j}(t)
\end{aligned}
$$

where $\tau_{1 m}$ represents the reference delay associated with backscatter from target traveling via path $m$ to element 1 (the reference element); and $\Delta \tau_{1 m}$ is the interelement delay associated with path $m$ originating from target in excess of $\tau_{1 m}$ with respect to the receiving element $j$.

The received signals of element $j$ is generalized to other array elements and expressed in the matrix form as

$$
Y(t)=(C \odot A) D X_{k} F(t)+V(t)
$$

where the symbol " $\odot$ " is Hadamard Product or Basic Product.; $Y(t)=\left[y_{1}(t), \cdots, y_{P}(t)\right]^{T}$ is defined as the received signal matrix received by the element $1-P$ of the SRA; $C$ is a $(P \times M)$ matrix representing the attenuation matrix with respect to the channel response function between the target and SRA elements, whose element $c_{j m}$ indicates the attenuation associated with backscatter from target traveling via path $m$ to element $j ; A=\left[a\left(\theta_{1}\right), a\left(\theta_{2}\right), \cdots, a\left(\theta_{M}\right)\right]$ is a $(P \times M)$ matrix representing a relative delay matrix to the reference element (element 1$)$, and is referred as the array steering matrix as shown by (5); $D=\left[e^{-j \omega_{c} \tau_{11}}, e^{-j \omega_{c} \tau_{12}}, \cdots, e^{-j \omega_{c} \tau_{1 M}}\right]^{T}$ is the reference delay matrix including interrelated delay from target traveling via all $M$ paths to element $1 ; F(t)=s(t) e^{j \omega_{c} t}$ is called the transmitting matrix; $V(t)$ indicates the noise matrix; and $X_{k}=\sum_{n=1}^{N} c_{k n} e^{-j \omega_{c} \tau_{k n}}=(C(k,:) \odot A(k,:)) D$ can be considered as a target-received signal matrix which is transmitted from the element $j$ through the channel to the target. 


$$
\begin{aligned}
A & =\left[a\left(\theta_{1}\right), a\left(\theta_{2}\right), \cdots, a\left(\theta_{M}\right)\right] \\
& =\left[\begin{array}{cccc}
e^{-j \omega_{c} \Delta \tau_{11}} & e^{-j \omega_{c} \Delta \tau_{12}} & \cdots & e^{-j \omega_{c} \Delta \tau_{1 M}} \\
e^{-j \omega_{c} \Delta \tau_{21}} & e^{-j \omega_{c} \Delta \tau_{22}} & \cdots & e^{-j \omega_{c} \Delta \tau_{2 M}} \\
\vdots & \vdots & \vdots & \vdots \\
e^{-j \omega_{c} \Delta \tau_{P 1}} & e^{-j \omega_{c} \Delta \tau_{P 2}} & \cdots & e^{-j \omega_{c} \Delta \tau_{P M}}
\end{array}\right] \\
& =\left[\begin{array}{cccc}
1 & 1 & \cdots & 1 \\
e^{-j \frac{2 \pi}{\lambda} d \sin \theta_{1}} & e^{-j \frac{2 \pi}{\lambda} d \sin \theta_{2}} & \cdots & e^{-j \frac{2 \pi}{\lambda} d \sin \theta_{M}} \\
\vdots & \vdots & \vdots & \vdots \\
e^{-j \frac{2 \pi}{\lambda}(P-1) d \sin \theta_{1}} & e^{-j \frac{2 \pi}{\lambda}(P-1) d \sin \theta_{2}} & \cdots & e^{-j \frac{2 \pi}{\lambda}(P-1) d \sin \theta_{M}}
\end{array}\right]
\end{aligned}
$$

\section{ADVTR Multipath DOA Model for ULA}

According to the idea of ADVTR, the first process of ADVTR is active detection, so the frequency of the transmitted signal has been known when the array receives the signal. Then the filter can be introduced to eliminate the impact of noise before TR operation. Subsequently VTR is performed virtually for the received signals of each SRA in the computer, and taking element $j$ as an example:

$$
y_{j}(-t)=B \cdot s(-t) e^{-j \omega_{c} t}=B f(-t)
$$

Where $B$ is expressed as equation (7), whose value is independent of time $t$ and can be considered as a coefficient.

$$
B=\left(\sum_{m=1}^{M} c_{j m} e^{-j \omega_{c} \Delta \tau_{j m}} e^{-j \omega_{c} \tau_{1 m}}\right) \cdot \sum_{n=1}^{N} c_{k n} e^{-j \omega_{c} \tau_{k n}}
$$

The signal is energy normalized and then re-transmitted virtually into the channel as a secondary transmission signal in the computer, which still satisfies the far field and narrow band model theory. Repeat the above first procedure, refer to (1) - (3), and suppose the signal received by element $l$ is $Z_{l}(t)$ :

$$
\begin{aligned}
z_{l}(t) & \approx \sum_{m=1}^{M} \sum_{n=1}^{N} c_{l m} c_{j n} g_{j} B s(-t) e^{-j \omega_{c}\left(t-\tau_{j n}-\tau_{1 m}-\Delta \tau_{l m}\right)} \\
& =\sum_{m=1}^{M} c_{l m} e^{j \omega_{c} \Delta \tau_{l m}} e^{j \omega_{c} \tau_{1 m}} \cdot \sum_{n=1}^{N} c_{j n} e^{j \omega_{c} \tau_{j n}} \cdot g_{j} B s(-t) e^{-j \omega_{c} t}
\end{aligned}
$$

Where $g_{j}$ is the normalized coefficient of element $j$ obtained with reference to the transmitted signal power, and owing to the principle of virtual time reversal, the secondary process has no noise.

Referring to (4) - (5), the received signal of all of elements of SRA on the second time with element $j$ probing virtually is

$$
Z_{j}(t)=\left(C \odot A^{*}\right) D^{*} X_{j}^{*} g_{j} y_{j}(-t)
$$

where $A, C, D$ are the same as the parameters of (4), and $X_{j}$ is the same as $X_{k}$ of (4) except that $X_{k}$ is the received signal matrix with element $k$ probing, $X_{j}$ is the received signal matrix with element $j$ probing.

The received signals element $j$ probing is generalized to other array elements and the sum of received signals of SRA after TR can be obtained:

$$
\begin{aligned}
Z t r & =\sum_{j=1}^{P} Z_{j}(t) \\
& =\sum_{j=1}^{P}\left(C \odot A^{*}\right) D^{*} X_{j}^{*} g_{j} y_{j}(-t) \\
& =\left(C \odot A^{*}\right) D^{*} \sum_{j=1}^{P} X_{j}^{*} g_{j} y_{j}(-t)
\end{aligned}
$$

\section{DOA Estimation AlgoRIthM}

\section{A. Conventional Multipath Capon Algorithm}

According to the array signal processing theory, the purpose of the Capon algorithm is attempting to minimize the power by noise and other interference signal except the desired signal, while maintaining a fixed gain in the direction from which the signal originated [6]. Combining the model established by (4), the Capon algorithm can be represented as the following minimum problem:

$$
\begin{aligned}
& \min w^{H}(\theta) \hat{R}_{Y} w(\theta) \\
& \text { S.T.: } w^{H}(\theta) a\left(\theta_{1}\right)=1
\end{aligned}
$$

Where $\hat{R}_{Y}=\frac{1}{N} \mathrm{E}\left\{\mathrm{Y}(\mathrm{t}) \mathrm{Y}(t)^{H}\right\}$ is the sample covariance matrix of the array output signal with the multipath; $\theta_{1}$ is desired angle information and $w$ is the weight value.

The Lagrange multiplier method can be used to obtain the solution of the above problem:

$$
w=\frac{\hat{R}_{Y}^{-1} a\left(\theta_{1}\right)}{a^{H}\left(\theta_{1}\right) \hat{R}_{Y}^{-1} a\left(\theta_{1}\right)}
$$

Equation (13) can be obtained by taking (12) into (11), which means the largest power in the desired direction while the power except the desired direction is the smallest:

$$
P=\frac{1}{a^{H}\left(\theta_{1}\right) \hat{R}_{Y}^{-1} a\left(\theta_{1}\right)}
$$

Thus the spatial spectrum of the Capon algorithm can be defined as:

$$
P=\frac{1}{a^{H}(\theta) \hat{R}_{Y}^{-1} a(\theta)}
$$

Where $a(\theta)$ is the steering vector and defined previously in (5) as: 


$$
a(\theta)=\left[1, e^{-j \frac{2 \pi}{\lambda} d \sin \theta}, \cdots, e^{-j \frac{2 \pi}{\lambda}(P-1) d \sin \theta}\right]^{T}
$$

\section{B. ADVTR Capon Algorithm}

According to (10), follow the same steps as the above conventional DOA estimation:

$$
\begin{aligned}
& \min w_{t r}{ }^{H}(\theta) \hat{R}_{z t r} w_{t r}(\theta) \\
& \text { subject to: } w_{t r}{ }^{H}(\theta) a_{t r}\left(\theta_{1}\right)=1
\end{aligned}
$$

Where $\hat{R}_{Z t r}=\frac{1}{N} \mathrm{E}\left\{Z \operatorname{tr}(\mathrm{t}) \mathrm{Ztr}(t)^{H}\right\}$ is the sample covariance matrix after virtual time reversal output signal and the other values are the same as above. At this time, the spatial spectrum of the ADVTR Capon algorithm is:

$$
P t r=\frac{1}{a_{t r}^{H}(\theta) \hat{R}_{\mathrm{Ztr}}{ }^{-1} a_{t r}(\theta)}
$$

Where $a_{t r}(\theta)$ is:

$$
a_{t r}(\theta)=\left[1, e^{j \frac{2 \pi}{\lambda} d \sin \theta}, \cdots, e^{j \frac{2 \pi}{\lambda}(P-1) d \sin \theta}\right]^{T}
$$

\section{SimUlation RESUlts}

The simulation environment is as follows: shallow sea waveguide, uniform sound speed environment, regardless of the impact of ocean additive noise, and the case of single objective. The simulation model is shown in Figure 1, where the number of TRM (SRA) is 9, the interelement spacing is $0.75 \mathrm{~m}$, the element 1 is $75 \mathrm{~m}$ from the sea surface, the depth of bottom is $500 \mathrm{~m}$, the sound speed is $1.5 \mathrm{~km} / \mathrm{s}$ and the range between PS and the target is $2 \mathrm{~km}$. The target is located in $253 \mathrm{~m}$, that is the DOA is $-5^{0}$. PS transmits $1 \mathrm{kHz}$ continuous wave $(\mathrm{CW})$ signal. The simulation environment is shown in Table 1.

\section{TABLE I. SIMULATION ENVIRONMENT}

\begin{tabular}{|c|c|}
\hline \multicolumn{1}{|c|}{ Simulation Condition } & Parameter \\
\hline Number of elements & 9 \\
\hline Interelement spacing & $0.75 \mathrm{~m}$ \\
\hline The depth of element 1 & $75 \mathrm{~m}$ \\
\hline The depth of bottom & $500 \mathrm{~m}$ \\
\hline The source depth & $78 \mathrm{~m}$ \\
\hline Target depth(-5 $\left.{ }^{0}\right)$ & $253 \mathrm{~m}$ \\
\hline The range & $2 \mathrm{~km}$ \\
\hline Number of multipath & 3 \\
\hline
\end{tabular}

Bellhop special acoustic field simulation toolbox under MATLAB simulation environment is used to simulate the ocean sound field environment. The multiple reflection paths by sea surface or bottom are ignored, and only the three paths illustrated in Figure 1 are considered. Then the various parameters used for simulation are obtained, as shown in Table 2. Because through the simulation, we can discover that the amplitudes of the same paths from the target to each element (i.e. the amplitude from the target to each element via the top-reflection) are very closer. Then for convenience, the amplitude information between PS and the target expressed in the table is the corresponding direct-path, top-reflection-path and bottom-reflection-path successively.

\section{TABLE II. SIMULATION PARAMETERS TARGET AT $-5^{0}$}

\begin{tabular}{|c|c|}
\hline Simulation Parameter & Value \\
\hline Direction of arrival & $\left\{4.458^{0}, 0^{0},-22.884^{0}\right\}$ \\
\hline Amplitude & $\{4.99 \mathrm{e}-4,5.0 \mathrm{e}-4,2.92 \mathrm{e}-4\}$ \\
\hline Delay of element 1 & $\{1.3372291,1.3333348,1.4479731\} \mathrm{s}$ \\
\hline
\end{tabular}

Figure 2 (a)-(d) shows the DOA estimation performance comparison with Capon estimator and ADVTR Capon estimator for target at $-5^{0}$ when the SNR is $0 \mathrm{~dB},-10 \mathrm{~dB},-15 \mathrm{~dB}$ and $-20 \mathrm{~dB}$.

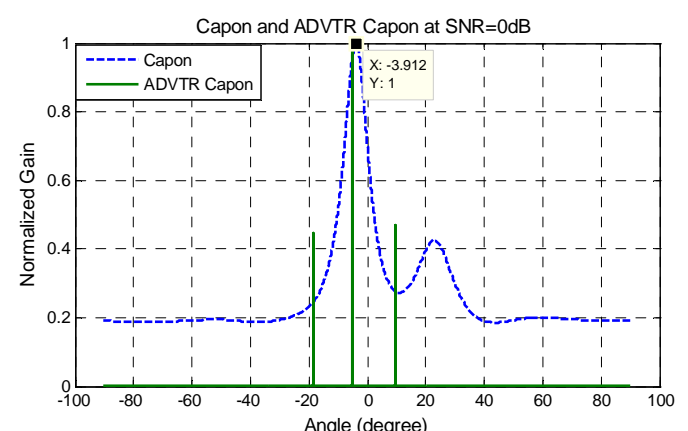

(a)

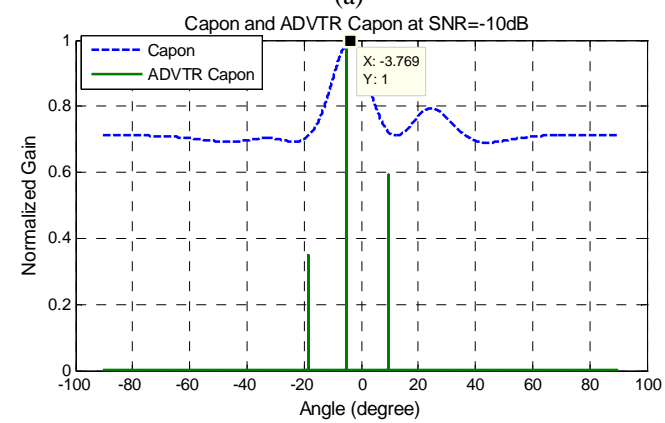

(b)

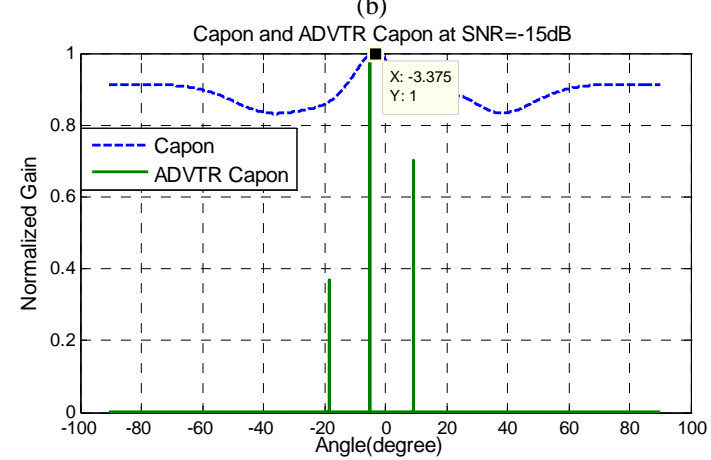

(c) 


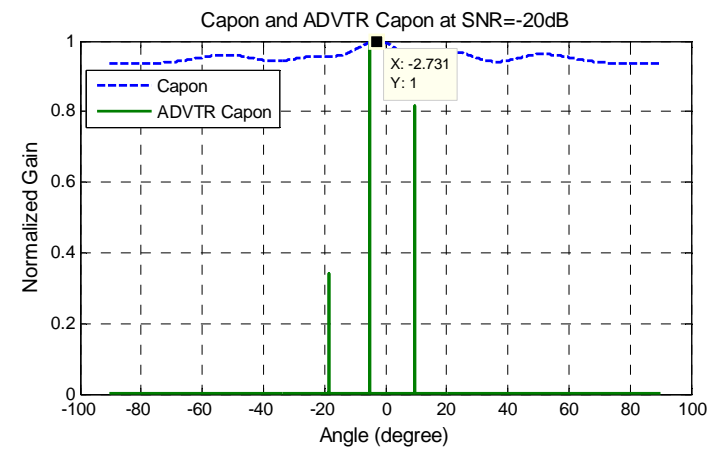

(d)

FIGURE II. CAPON ESTIMATOR AND ADVTR CAPON ESTIMATOR AT $\theta=-5^{\circ}$ : (a) $\mathrm{SNR}=0 \mathrm{~dB}$; (b) $\mathrm{SNR}=-10 \mathrm{~dB}$; (c) $\mathrm{SNR}=-15 \mathrm{~dB}$; (d) $\mathrm{SNR}=-20 \mathrm{~dB}$

It can be seen from Figure 2 that:

(1) Relative to Capon algorithm, ADVTR Capon algorithm can estimate accurately the expected value of the target, whose energy of the main lobe is far higher than its corresponding sidelobes and resolution is higher.

(2) With the changes of SNR from $0 \mathrm{~dB}$ to $-20 \mathrm{~dB}$, the relations between main lobe and sidelobes for both Capon and ADVTR Capon algorithm are the same that the energy of the sidelobes is higher and higher, and is getting closer to the main lobe. The difference is that the resolution for Capon algorithm is getting lower and lower, and the estimation deviation is more and more great. However, the resolution for ADVTR Capon is almost not affected, and the target angle can be estimated without bias.

\section{CONCLUSION}

In this paper, we use the ADVTR method to study the performance of underwater target DOA in the presence of multipath, and a DOA estimation algorithm based on ADVTR Capon is proposed. Firstly, based on the ray theory, a conventional multipath DOA estimation model and an ADVTR DOA estimation model for ULA are established, and then the model is estimated by Capon algorithm. The simulation results show that the DOA estimator with ADVTR, compared with the conventional method without TR, has a more accurately estimated value, a higher resolution and a stronger ability to suppress sidelobes, so that the target DOA estimation can be achieved in the case of low SNR and multipath.

\section{ACKNOWLEDGMENT}

This work was supported by National Natural Science Foundation of China (61571365, 61571367, and 61671386), National Key R\&D Program of China (2016YFC1400200), and Scientific Research Project of Shaanxi Provincial Education Department (16JK2173).

\section{REFERENCES}

[1] H. Krim and M. Viberg, "Two decades of array signal processing research: the parametric approach,” IEEE Signal Processing Magazine, vol. 13, no. 4, pp. 67-94, July 1996.
[2] E. Daeipour,W. D. Blair, and Y. Bar-Shalom, "Bias compensation and tracking with monopulse radars in the presence of multipath," IEEE Trans. Aerosp. Electron. Syst., vol. 33, no. 3, pp. 863-882, July 1997.

[3] Mathias Fink, “Time reversal of ultrasonic fields-Part I: Basic principles,” IEEE Trans. Ultras., Ferroelec., Freq. Contr., vol.3, no.5, pp.555-566, September 1992.

[4] Hee-Chun Song,“An Overview of Underwater Time-Reversal Communication,” IEEE Journal of oceaninc engineering, vol. 41, no. 3, July 2016.

[5] A. Silva, S. Jesus, J. Gomes, and V. Barroso, "Underwater acoustic communication using a virtual electronic time-reversal mirror approach," in Proc. 5th Eur. Conf. Underwater Acoust., Lyon, France,Jun. 2000, vol. V.

[6] Foroohar Foroozan, Amir Asif, “Time Reversal Based Active Array Source localization,” IEEE Trans. SP, vol. 59, no. 6, pp. 2655-2668, June 2011.

[7] Yongqing Fu,Wei Liu, Ruijie Bai, Jingrui Li, and JinlinWang, “ A Novel Virtual Time Reversal Method for Passive Direction of Arrival Estimation," Mathematical Problems in Engineering, Volume 2015, Article ID 613692, 12 pages, http://dx.doi.org/10.1155/2015/613692.

[8] Xiaolu Zeng, Minglei Yang, Baixiao Chen and Yuanwei Jin, "Low Angle Direction of Arrival Estimation by Time Reversal,” 2017 IEEE International Conference on Acoustics, Speech and Signal Processing (ICASSP), pp. 3161-3165, New Orleans, LA, USA, March 2017.

[9] Fouzhou Yang, Haiyan Wang, Xiaohong Shen and Haixia Jing, "Superdirection element distribution model of NLA based on TR," Journal of Shanghai Jiao Tong University, vol. 47, no. 12,pp. 1907-1910, December 2013. 